\title{
POSTER
}

\section{Apport du sialo-conebeam en pathologie salivaire obstructive non tumorale}

\section{Delebarre H, Cochais P, Santini J, Afota F, Savoldelli C}

L'imagerie par faisceau conique (CBCT) était initialement indiquée dans l'étude des tissus osseux et dentaires. Depuis 2009, elle occupe désormais un intérêt grandissant dans l'exploration des glandes salivaires principales parotidiennes et sous maxillaire. Cet examen, simple de réalisation, offre la possibilité d'étudier les canaux salivaires en 3 dimensions à la recherche de sténose canalaire ou d'obstruction lithiasique. La précision de la localisation de l'obstruction du sialo-conebeam rend cet examen compétitif face à l'échographie, scanner, IRM et sialographie conventionnelle. Nous exposons le protocole de mise en oeuvre au CSD du CHU de Nice de cet examen ainsi que les résultats attendus pour améliorer la planification de la prise en charge thérapeutique de ces pathologies. 